\title{
Avian influenza vaccination in Egypt: Limitations of the current strategy
}

\author{
Marisa Peyre ${ }^{1,3^{*}}$, Hamid Samaha ${ }^{2}$, Yilma Jobre Makonnen ${ }^{3}$, Ahmed Saad ${ }^{3}$, Amira Abd-Elnabi ${ }^{2}$,
} Saber Galal $^{2}$, Toni Ettel ${ }^{3}$, Gwenaelle Dauphin ${ }^{4}$, Juan Lubroth ${ }^{4}$, François Roger ${ }^{1}$, Joseph Domenech ${ }^{4}$

${ }^{1}$ French Agricultural Research Center for International Development (CIRAD), Animal and Integrated Risk Management Research Unit, Baillarguet Campus, 34398 Montpellier Cedex 5, France, ${ }^{2}$ General Organisation for Veterinary Services, Cairo, Egypt, ${ }^{3}$ Emergency Center for Transboundary Animal Diseases (ECTAD), Food and Agriculture Organisation of the United Nations (FAO), Cairo, Egypt, ${ }^{4}$ Food and Agriculture Organisation of the United Nations (FAO) Viale delle Terme di Caracalla, Rome, Italy

*Correspondence to: Marisa Peyre, Email: marisa.peyre@cirad.fr, Tel: +33 467593868

Received 31 August 2009, Revised 12 November 2009, Accepted 16 November 2009, Published online 09 December 2009

J Mol Genet Med (2009), 3(2), 198-204

(C) Copyright The Authors: This is an open access article, published under the terms of the Creative Commons Attribution Non-Commercial License (http://creativecommons.org/licenses/by-nc/2.0/uk/). This license permits noncommercial use, distribution and reproduction of the article, provided the original work is appropriately acknowledged with correct citation details.

\begin{abstract}
Vaccination of domestic poultry against avian influenza (AI) has been used on a large-scale in South East Asia since 2003 and in Egypt since 2006 to fight H5N1 highly-pathogenic avian influenza (HPAI) epidemics. The decision to use mass vaccination against HPAI in Egypt was taken as an emergency measure based on positive impact of such control measures in Vietnam and the People's Republic of China. However, three years on, the impact on disease control of AI vaccination in Egypt has been very limited. Despite the continuous vaccination of poultry against HPAI, poultry outbreaks and human cases are reported regularly. A recent assessment study highlighted substantial weaknesses in the current immunisation programme and its lack of positive impact on the spread of infection or the maintenance of public health safety. The shortcomings of the vaccination strategy may be attributed in part to a lack of sufficient support in terms of funding and communication, the absence of an efficient monitoring system, and inadequate training of field technicians. The difficulties of blanket vaccinations in semi-commercial farms and household poultry sectors are well known, however, improvements in the industrial sector should be possible though better government controls and greater collaboration with the private sector. AI vaccination should be regarded as just one control tool within a broader disease control program integrating surveillance, outbreak investigation, disease management systems, and the rigorous implementation of bio-security measures. If incorrectly implemented, AI vaccination has a limited impact as a disease control measure. Moreover, without strict bio-security precautions undertaken during its application, farm visits to vaccinate poultry could facilitate the spread of the virus and therefore become a risk factor with important implications on the maintenance of the virus and potential risk for human exposure.
\end{abstract}

KEYWORDS: Avian influenza, disease control, vaccination strategy, public health risk, Egypt

\section{INTRODUCTION}

Vaccination could be a useful tool in controlling avian influenza (AI) outbreaks. However, a carefully conceived vaccination strategy must be accompanied by strict biosecurity measures and efficient monitoring systems.
Countries which have authorized vaccination to control avian influenza outbreaks under special circumstances in the past have met with contradictory results, from effective control and disease eradication to antigenic drift of the viral strain (Lee et al, 2004; Busani et al, 2009; Capua et al, 2009). Extensive vaccination programs are currently 
ongoing in South East Asia and Egypt to control the H5N1 HPAI epidemics (Peyre et al, 2009; Domenech et al, 2009). Some of these countries have succeeded in reducing HPAI incidence and thereby reducing human risk of infection (e.g., Vietnam and China). Vietnam has managed to bring the disease under control within two years by strictly adhering to vaccination guidelines and implementing efficient outbreak management (To et al, 2007; Domenech et al, 2009). SAR Hong Kong was also able to control and even eradicate the H5N1 HPAI virus by using mass vaccination accompanied by high standard bio-security measures and efficient post-vaccination monitoring (Ellis et al, 2006). In contrast, countries which have implemented mass vaccination without extensive outbreak management and bio-security measures are still fighting to control the infection (e.g., Indonesia and Egypt) (Domenech et al, 2009; EMPRES/GLEWS, 2009). Vaccination does not confer complete sterilising immunity and some vaccinated birds may continue to be infected and hence be contagious. If not monitored properly, the virus can circulate silently within a vaccinated flock (Hulse-Post et al, 2005; Savill et al, 2006). Therefore vaccination must be integrated within a broader control program that includes outbreak management and efficient surveillance and monitoring systems.

H5N1 HPAI virus appears to have emerged in Egypt in early 2006. During the first wave of outbreaks, only stamping-out (within $3 \mathrm{~km}$ of the initial outbreak), quarantine surveillance, and some movement control (within a $7 \mathrm{~km}$ radius from the outbreak location) measures were implemented in an attempt to contain the disease, achieving only limited success. Initially, emergency vaccination was used to protect grand-parent and parent flocks. However, the disease spread to most Governorates within a few weeks and the country was declared endemic in July 2008. The veterinary authorities considered vaccination to be the most efficient tool in controlling this infectious disease. At this stage (March 2006), the decision was taken to vaccinate all commercial flocks, followed by vaccination of the household poultry, starting in May 2007.
Three years later, the implementation of AI vaccination in Egypt has had a limited impact on disease control. Poultry outbreaks and human cases continue to be reported regularly (Table 1) (EMPRES/GLEWS, 2009). However, it is difficult to predict how the situation would have evolved if there had been no vaccination at all. A recent assessment study performed by FAO has highlighted substantial limitations in current immunisation practices, which have not curbed the spread of infection nor improved public health safety (Peyre, 2009). Among the possible explanatory factors are the limited incentives given to the veterinarian service staff carrying out vaccinations in the field and a lack of confidence and cooperation on the part of local communities. This report reviews the organisation and implementation of Egypt's AI vaccination strategy and examines the factors behind its limited control of the disease and its impact on public health.

\section{EGYPTIAN AI VACCINATION STRATEGY}

AI vaccination in Egypt has been the main tool used over the past three years to control the H5N1 HPAI epidemic. The focus on vaccination appears to have distracted attention away from other essential/critical procedures and disease control measures such as surveillance, bio-security outbreak investigation, and disease management interventions. Indeed, more than 80\% (24 million USD) of the available budget for HPAI control has been devoted to vaccination since the programme was launched (GOVS, 2009).

The national vaccination program for household poultry is organised by the government and vaccines are provided free-of-cost to local communities (Table 2). This program also covers farms with up to 500 birds. Six-monthly vaccination campaigns are carried out with some vaccination of "residuals" in between campaigns (birds which have not been vaccinated for various reasons, e.g., owners not on site during the visit; birds not presented to vaccinators, etc). Birds are vaccinated following a door-todoor protocol with the vaccination team entering each house to vaccinate rooftop birds.

Table 1: Number of annual HPAI outbreaks in commercial poultry farms and household poultry sector in Egypt (data provided by GOVS).

\begin{tabular}{|c|c|c|c|c|c|c|c|c|c|}
\hline \multirow{2}{*}{ Year } & \multicolumn{4}{|c|}{ Commercial poultry farms } & \multicolumn{3}{c|}{ Household poultry } & Total \\
\cline { 2 - 10 } & Vaccinated & $\begin{array}{c}\text { Non- } \\
\text { vaccinated }\end{array}$ & Unknown & $\begin{array}{c}\text { Sub- } \\
\text { total }\end{array}$ & Vaccinated & $\begin{array}{c}\text { Non- } \\
\text { vaccinated }\end{array}$ & Unknown & $\begin{array}{c}\text { Sub- } \\
\text { total }\end{array}$ & (2) \\
\hline $\mathbf{2 0 0 6}$ & - & - & 1318 & 1318 & 4 & 2 & 317 & 323 & $\mathbf{1 6 4 1}$ \\
\hline $\mathbf{2 0 0 7}$ & 26 & 3 & 5 & 34 & 36 & 79 & 162 & 277 & $\mathbf{3 1 1}$ \\
\hline $\mathbf{2 0 0 8}$ & 22 & 2 & 2 & 26 & 25 & 22 & 42 & 89 & $\mathbf{1 1 5}$ \\
\hline $\mathbf{2 0 0 9}$ & 5 & 1 & 10 & 16 & 4 & 2 & 77 & 83 & $\mathbf{9 9}$ \\
\hline Total & 53 & 6 & 1335 & 1394 & 69 & 105 & 598 & 772 & $\mathbf{2 1 6 6}$ \\
\hline
\end{tabular}


Table 2: Main features of the AI vaccination strategy conducted by GOVS in household poultry in Egypt and its limitations.

\begin{tabular}{|l|l|}
\hline AI vaccination strategy in household poultry & Limitations \\
\hline $\begin{array}{l}2 \text { campaigns per year, each one lasting 3 months } \\
\text { Door-to-door vaccination protocol }\end{array}$ & $\begin{array}{l}\text { Limited coverage with a maximum of 36\% of the total bird } \\
\text { population being vaccinated. } \\
\text { Risk for mechanical transfer of the virus with limited bio- } \\
\text { security precautions. }\end{array}$ \\
\hline $\begin{array}{l}\text { Only one dose of vaccine is administered for all birds (no } \\
\text { booster) }\end{array}$ & $\begin{array}{l}\text { Booster doses could be required with inactivated vaccines } \\
\text { to confer long term protection and limit contact } \\
\text { transmission especially in groups of mix ages and species }\end{array}$ \\
\hline Chicks and ducklings are vaccinated & $\begin{array}{l}\text { Inactivated vaccines are not efficient in day old birds and } \\
\text { vaccination at a young age could impair adult immunity }\end{array}$ \\
\hline $\begin{array}{l}\text { In some Governorates, double or more volume dose of vaccine } \\
\text { is given to ducks and geese }\end{array}$ & $\begin{array}{l}\text { Vaccination protocols are not harmonised within the } \\
\text { country as there are no standard operating procedures in } \\
\text { place; veterinarians tend to follow the instructions indicated } \\
\text { on the vaccine bottle or technical sheet. }\end{array}$ \\
\hline
\end{tabular}

Commercial farmers must assume the entire cost of vaccinations on their farms (farms with more than 500 birds including sector 3 farms) and they are responsible for defining their own vaccination programs, including the choice of vaccine and vaccination protocols (Table 3 ). Due to limited supervision of public veterinary services (monitoring less than $6.5 \%$ of the farms), there is no clear information on the AI vaccination practices and the scale of operations in the commercial sector.

All imported AI vaccines are evaluated by the Central Laboratory for Evaluation of Veterinary Biologicals (CLEVB, certified ISO 17025) before being released to the market. AI vaccine evaluation is performed using international standards of quality insurance; the methods used include purity/quality, safety, sterility, and potency tests. The latter involves immunogenicity response in SPF chickens and challenge test against Egyptian isolates (in use for batch release since March 2009).

There is no post-vaccination monitoring system in place for household poultry (Table 2). There is a surveillance protocol in place for commercial poultry farms $(6.5 \%$ of the farms voluntarily submit samples for post-vaccination monitoring) (Table 3).

\section{IMPACT OF THE CURRENT VACCINATION STRATEGY}

\section{Impact on disease control and surveillance}

Due to the practical difficulties involved in vaccinating household poultry in the field, the vaccine coverage is very low, with an average of $25-30 \%$ in the best vaccinated Governorates while dropping to as little as 1\% in some villages (Rijks and ElMasry, 2009). The impact of the AI vaccination strategy on the control of the disease therefore is conspicuously limited. However, in villages where coverage has reached at least $50 \%$, a lower H5N1 HPAI incidence rate has been observed (GOVS, personal communication).
At the level of commercial farms, around $50-60 \%$ are thought to be vaccinating against AI (GOVS, personal communication). This higher level of coverage may be the reason why AI vaccination appears to be having a more positive impact in the commercial sector compared to the household poultry sector, with an important decrease in outbreaks reported in the years following the implementation of vaccination (Table 1). However, a proper impact study needs to be carried out to confirm these observations, as there may be considerable underreporting of the infection incidents (GOVS, personal communication).

The current strategy for AI vaccination of household poultry in Egypt probably has become a risk factor in the spread of the infection due to: i) Limited understanding and use of bio-security precautions, for example, vaccinators do not use overshoes correctly, often wearing them inside their shoes to protect their feet and consequently failing to prevent potential mechanical transmission of viruses between houses during the door-todoor vaccination campaigns (Figure 1A): ii) A lack of means to disinfect and clean, or dispose-off spoiled and contaminated needles between houses while vaccinating sick birds (Figures 1B): iii) The use of emergency ring vaccination as the first action following the declaration of an outbreak, starting with the remaining birds within the infected villages and moving out to neighbouring villages. On some occasions, vaccinations have been carried out even before proper outbreak management procedures, such as safe culling, the disposal of carcasses, and the disinfection of the premises, have been implemented.

The efficacy of the vaccines currently in use in Egypt has been criticized due to the occurrence of numerous outbreaks within vaccinated villages. However, given the vaccine quality controls in place, this phenomenon probably relates more to the improper use of the vaccines (which would impair the level of immunity) rather than the efficacy of the vaccine itself. Indeed, while it has been 
Table 3. Main features of the AI vaccination strategy conducted in commercial poultry in Egypt and its limitations.

\begin{tabular}{|l|l|}
\hline AI vaccination strategy in commercial farms & Limitations \\
\hline $\begin{array}{l}\text { One dose is administered to broilers and multiple doses to } \\
\text { layers and breeders }\end{array}$ & $\begin{array}{l}\text { Vaccination protocol varies from farm to farm as no proper } \\
\text { standardised protocols are in place, most of sector } 3 \text { farms } \\
\text { do not vaccinate the broilers }\end{array}$ \\
\hline Day old chicks are vaccinated in hatcheries & $\begin{array}{l}\text { Inactivated vaccines are not efficient in day old birds and } \\
\text { vaccination at a young age could impair adult immunity }\end{array}$ \\
\hline $\begin{array}{l}\text { Most of duck and geese farms are not applying HPAI } \\
\text { vaccination }\end{array}$ & $\begin{array}{l}\text { Without any regular monitoring, there is a risk of the silent } \\
\text { circulation of the field virus in the environment (ducks and } \\
\text { geese usually are asymptotic carriers) }\end{array}$ \\
\hline $\begin{array}{l}\text { Some post-vaccination monitoring is performed by private } \\
\text { laboratories }\end{array}$ & $\begin{array}{l}\text { No information is available on vaccination coverage and } \\
\text { effectiveness in farms }\end{array}$ \\
\hline $\begin{array}{l}\text { National monitoring is done on a volunteer basis which } \\
\text { represents only } 6.5 \% \text { of total farms }\end{array}$ & $\begin{array}{l}\text { There is an increasing risk of the silent circulation of the } \\
\text { field virus within vaccinated flocks and the spread of the } \\
\text { infection to the household sector if the birds are sold to } \\
\text { local markets }\end{array}$ \\
\hline
\end{tabular}

demonstrated that inactivated vaccines requires booster doses to confer mid-term protective level of immunity (up to 4 months) (Unger et al, 2008; Peyre et al, 2009), a single dose vaccination protocol currently is enforced in household poultry in Egypt. One-day old chicks are vaccinated with inactivated vaccines, although clinical trials conducted in Indonesia (Unger et al, 2008) have shown that this practice is inefficient and may impair adult immunity, thereby limiting the efficacy of subsequent vaccination. Egyptian ducks and geese also are either not vaccinated or given the same dose as chickens, which is not sufficient to confer protective immunity in these species (Tian et al, 2005). Finally, current inactivated vaccines may only confer limited protection in local Egyptian avian breeds; no study has yet been carried out on the efficacy of vaccines in local birds in the field (Sawitri et al, 2007). An FAO research project is currently ongoing to address both the issue of current vaccine efficacy in the field (in local breeds) and against all local strains of circulating viruses in Egypt (Avian Influenza Vaccine Efficacy Project Egypt (AIVEP)).

The apparent reported decrease in disease incidence, which has been observed since the use of mass vaccination in Egypt (Table 1), may be the result of lower outbreak detection and inadequate reporting rather than immunity conferred by vaccination. Positive birds detected in live bird markets and data from participatory disease surveillance implemented as part of the SAIDR research project (Strengthening Avian Influenza Detecting and Response) (Rijks and ElMasry, 2009) have indicated the incidence of outbreaks on household poultry and commercial farms although farmers have not reported them.

This inadequate reporting could be due to:

-A lack of incentive for poultry owners to report outbreaks as there is no compensation scheme in place.
- A lack of knowledge and misconceptions regarding the effectiveness of vaccination on the part of decisionmakers and field veterinarians. Decision-makers tend to believe that AI vaccination provides $100 \%$ protection in all vaccinated birds and hence can prevent all outbreaks. Local veterinary services consequently are reluctant to declare new outbreaks due to the fear of being unfairly blamed for failing to effectively perform their duties. While vaccinations are occasionally performed improperly, such as vaccination in outbreak areas or in their immediate vicinity, at other times new outbreaks are a reflection of the failure to reach sufficient vaccine coverage.

Taken together, the limited vaccine efficacy of some vaccines against new viral isolates (Dr Elham, Director CLEVB, personal communication; Swayne and Kapczynski, 2008), a potentially lower protection in local breeds similar to what is being demonstrated in Indonesia (Sawitri et al, 2007; Unger et al, 2008), and no surveillance of circulating viruses in vaccinated areas have contributed to an under-reported circulation of the virus, which favours the persistency of the infection and increases the risks for bird to human transmission (GOVS, personal communication).

\section{Impact on public behaviour}

A false sense of security may be observed in some community members based on the belief that poultry vaccination can confer protection for human health. These individuals consequently do not feel the need to increase bio-security precautions when dealing with poultry or poultry products. At the same time, other community members are losing faith in government actions since numerous cases of sudden deaths in poultry have apparently followed poultry vaccination campaigns, leading to a loss of confidence in AI vaccination. This so called "post-vaccination sudden death" in fact may be explained by classic events that occur during vaccination 
with the improper use of vaccines (vaccines injected at $4^{\circ} \mathrm{C}$ or incorrect injection practices, spread of infection as no strict bio-security precautions are being followed during vaccination). The result is that poultry owners are becoming reluctant to vaccinate their birds.

However, the community would be more inclined to follow bio-security precautions when dealing with poultry if; i) veterinary services staff observed proper procedures in terms of bio-security precautions during vaccination campaigns, outbreak investigations, culling operations, and ii) conveyed correct message to the public when given the opportunity. In reality, messages are not efficiently delivered by field veterinarians due to their limited training in correct stamping out practices and the advantages of control measures such as AI vaccination. Consequently, poultry owners put their health at risk by not believing and not applying public health safety messages delivered through national communication campaigns.

\section{A:}

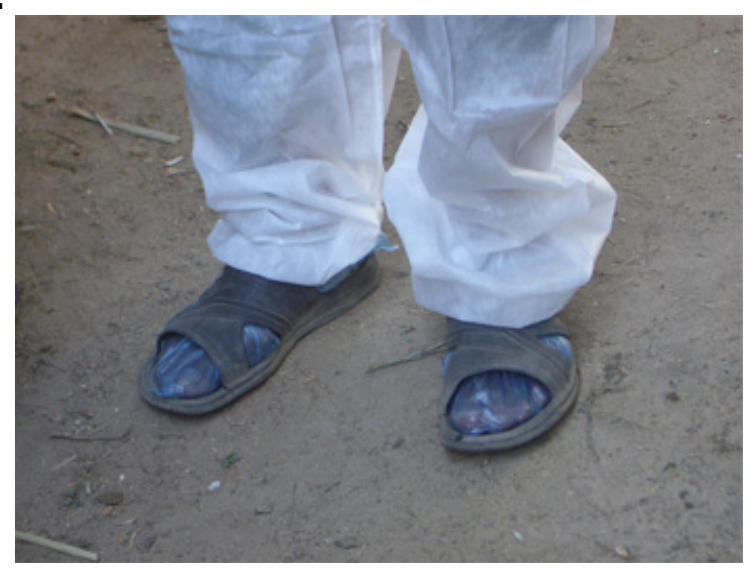

B:

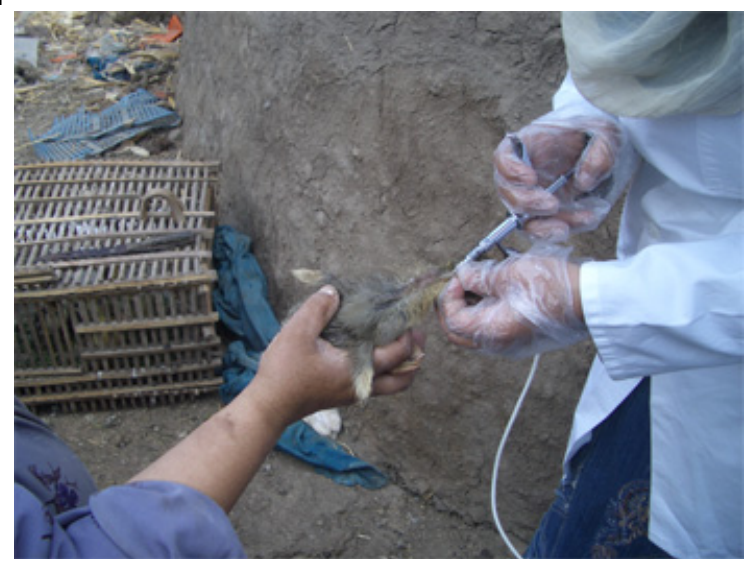

Figure 1. A. Improper use of personal protective equipment during household poultry vaccination campaign: the vaccinator is wearing overshoes inside his shoes to protect his feet. B. Improper use of AI vaccines during household poultry vaccination campaign: A sick duckling is being vaccinated with an automatic syringe; poultry in the next house will be vaccinated with the same equipment prior disinfection.

\section{Impact on potential human pandemic}

In Egypt, clade 2.2 viruses seem to have evolved and diverged significantly compared to other viruses belonging to the same clade in Africa, Europe and Asia as demonstrated by the emergence of a distinct third order clade (namely 2.2.1), which includes all the Egyptian isolates analysed so far (Cattoli et al, 2009; WHO/OIE/FAO H5N1 Evolution Working Group, 2009). However, it is difficult to determine without further investigations whether this is due to faster evolutionary rate of a distinct population of viruses, or the immune pressure of the vaccination strategy in place. There is evidence of increased virulence in birds and antigenic mutations away from the vaccine strains as some avian vaccines can no longer give full protection against viral mutants (Dr Elham, Director CLEVB, personal communication; Swayne and Kapczynski, 2008). However, there is no evidence that these genetic changes have any consequences on contagiousity or pathogenecity in humans. Attention should be paid to this potential effect and intensive monitoring of virus circulation should be set up for backyard and commercial systems.

The inadequate implementation of vaccination campaigns compromises disease control and can give the public an erroneous impression that the risk to humans is reduced.

\section{GENERAL RECOMMENDATIONS FOR CORRECT VACCINATION PRACTICES}

Vaccination against HPAI should be considered as just one of several tools in a comprehensive HPAI control package that should include the improvement of surveillance systems and the proper implementation of outbreak investigations, disease management, and bio-security measures. AI vaccination increases resistance of the host to the disease, but will not ensure the complete prevention of infection nor the total absence of contact transmission (Peyre et al, 2009). Moreover, complete protective immunity takes two weeks to develop and multiple doses are required to confer longterm protection (Peyre et al, 2009). The efficacy of AI vaccines also can be limited by the type of vaccine and its formulation (antigen content, adjuvant, viral strain) as well as age, species and type of breed of the targeted birds (Peyre et al, 2009). Soundly defined and integrated AI vaccination policies are required that include the definition of a national AI vaccination strategy, setting up the specific objectives of the vaccination and the action plan (vaccination program) to reach the objectives. The vaccination program should include detailed standard operating procedures for implementation (e.g., vaccination protocols - number and timing of the vaccine doses according to the poultry production type and bird species/breed), logistical planning, and routine evaluations and selection of vaccine according to the local context. Vaccination should not be performed during outbreaks or under limited bio-security precautions. Vaccination during outbreaks is not recommended because it not only fails to stop the infection of birds it probably contributes to propagating the infection and raises human health safety concerns.

Properly regulated vaccination monitoring under government control, covering the commercial as well as 
household sectors, is critical to ensure vaccination effectiveness and to monitor possible viral mutations in circulating viruses.

An AI vaccination strategy also should include training and education of technical staff on vaccination issues (biosecurity, vaccine administration, correct use and disposal of personal protective equipment, professional ethics) and an outreach programme to enhance community awareness and cooperation. These elements are critical in disease control/elimination interventions, the prevention of disease spread in the animal population, and in the reduction of human exposure.

Efficient awareness campaigns on bio-safety practices, including the safe-handling of birds for public health protection, are needed to increase cooperation between local populations and technical staff, and to limit public health risks, particularly the risk of infection for children (e.g., isolation of sick birds from the flock, preventing children from playing with suspect or dead birds).

It is likely that H5N1 HPAI will persist in Egypt for some time, as is currently the case in several Southeast Asian countries. All of the epidemiological factors that are maintaining the virus in the environment are not yet clearly understood although ongoing research projects are trying to improve understanding of this complex epidemiology. For economical as well as sociological reasons, mass vaccination cannot be continued on a longterm basis and exit strategies or more strategic, targeted vaccination need to be considered. Vaccination should only be implemented as part of an overall HPAI control package that includes other control measures such as proper stamping out, bio-security improvements, and the control of movements of animals and animal products. AI vaccination policy and strategies need to be reassessed periodically and operational plans revised according to their impact on disease incidence.

\section{CONCLUSIONS}

AI vaccination only should be implemented if certain conditions are met. These include the implementation of an efficient monitoring system and effective outreach programmes. The latter is essential to ensure community cooperation needed for high vaccination coverage. If this cannot be ensured, vaccination must not be considered to be a kind of miraculous tool that will allow the control and elimination of the disease.

\section{ACKNOWLEDGMENTS}

This work was carried out within the framework of the SAIDR (Strengthening Avian Influenza Detection and Response) project jointly implemented by GOVS and FAO ECTAD based in Cairo, Egypt and funded by the United State Agency for International Development (USAID). The publication of the work was supported by the Priority Solidarity Fund (FSP) GRIPAVI project funded by the French Ministry of Foreign and European Affairs (MAEE). The authors would like to acknowledge Dr G Cattoli, the National Laboratory for Quality control of Poultry products
(NLQP) and the Central Laboratory for Evaluation of Veterinary Biologicals (CLEVB) for their valuable inputs.

The views expressed in this information product are those of the author(s) and do not necessarily reflect the views of the Food and Agriculture Organization of the United Nations.

\section{COMPETING INTERESTS}

None declared.

\section{LIST OF ABBREVIATIONS}

AI: Avian influenza

CIRAD: Centre International de Recherche pour l'Agriculture et le Développement

CLEVB: Central Laboratory for Evaluation of Veterinary Biologicals

ECTAD: Emergency Center for Transboundary Animal Diseases EMPRES: Emergency Prevention System for Transboundary Animal Diseases

FAO: Food and Agriculture Organisation of the United Nations

GLEWS: Global Early Warning System for Major Animal Diseases

GOVS: General Organisation of Veterinary Services

HPAI: Highly Pathogenic Avian Influenza

LE: Egyptian Pound

MAE: French Ministry of Foreign and European Affairs

NLQP: National Laboratory for Quality control of Poultry products

SAIDR: Strengthening Avian Influenza Detection and Response SOPs: Standard Operating Procedures

USAID: United State Agency for International Development

\section{REFERENCES}

Busani L, Toson M, Stegeman A et al. 2009. Vaccination reduced the incidence of outbreaks of low pathogenicity avian influenza in northern Italy. Vaccine, 27, 3655-3661.

Capua I, Schmitz A, Jestin V, Koch G and Marangon S. 2009. Vaccination as a tool to combat introductions of notifiable avian influenza viruses in Europe, 2000 to 2006. Rev Sci Tech, 28, 245-259.

Cattoli G, Monne I, Fusaro A et al. 2009. Highly pathogenic avian influenza virus subtype $\mathrm{H} 5 \mathrm{~N} 1$ in Africa: a comprehensive phylogenetic analysis and molecular characterization of isolates. PLoS One, 4, e4842.

Domenech J, Dauphin G, Rushton J et al. 2009. Experiences with vaccination in countries endemically infected with highly pathogenic avian influenza: the Food and Agriculture Organization perspective. Rev Sci Tech, 28, 293-305.

Ellis TM, Sims LD, Wong HK et al. 2006. Use of avian influenza vaccination in Hong Kong. Dev Biol (Basel), 124, 133-143.

EMPRES/GLEWS. 2009. H5N1 HPAI Global Overview - April 2009. Food and Agriculture Organisation, Emergency Prevention Systems and Global Early Warning System for Major Animal Diseases HPAI bulletin. Issue No 10.

GOVS. 2009. Plan for controlling Avian Influenza. General Organisation of Veterinary Services and Ministry of Agriculture and Land Reclamation. Arab Republic of Egypt.

Hulse-Post DJ, Sturm-Ramirez KM, Humberd J et al. 2005. Role of domestic ducks in the propagation and biological evolution of highly pathogenic H5N1 influenza viruses in Asia. Proc Natl Acad Sci USA, 102, 10682-10687.

Lee CW, Senne DA and Suarez DL. 2004. Effect of vaccine use in the evolution of Mexican lineage H5N2 avian influenza virus. J Virol, 78, 8372-8381. 
Peyre M. 2009. Assessment of Highly Pathogenic Avian Influenza vaccination strategy in Egypt. Food and Agriculture Organisation report. OSRO/EGY/701/USA.

Peyre M, Fusheng G, Desvaux S and Roger F. 2009. Avian influenza vaccines: a practical review in relation to their application in the field with a focus on the Asian experience. Epidemiol Infect, 137, 1-21.

Rijks J and ElMasry I. 2009. Characteristics of poultry production in Egyptian villages and their effect on HPAI vaccination campaign results - Results of a participatory epidemiology study. Food and Agriculture Organisation report.

Savill NJ, St Rose SG, Keeling MJ and Woolhouse ME. 2006. Silent spread of H5N1 in vaccinated poultry. Nature, 442, 757.

Sawitri SE, Darminto, Weaver J and Bouma A. 2007. The vaccination programme in Indonesia. Dev Biol (Basel), 130, 151-158.

Swayne DE and Kapczynski D. 2008. Strategies and challenges for eliciting immunity against avian influenza virus in birds. Immunol Rev, 225, 314-331.
Tian G, Zhang S, Li, Y et al. 2005. Protective efficacy in chickens, geese and ducks of an H5N1-inactivated vaccine developed by reverse genetics. Virology, 341, 153-162.

To TL, Bui QA, Dau NH et al. 2007. Control of avian influenza: a vaccination approach in Viet Nam. Dev Biol (Basel), 130, 157-158.

Unger F, Mariner J, Bett B et al. 2008. Avian influenza control in Indonesia: a situation overview, pro poor orientated research approaches of ILRI targeting backyard poultry- preliminary results. In Tropentag 2008: "Competition for resources in changing world: new drive for rural development" conference proceedings. Honhenheim, 7-9 October.

WHO/OIE/FAO H5N1 Evolution Working Group. 2009. Continuing progress towards a unified nomenclature for the highly pathogenic H5N1 avian influenza viruses: divergence of clade 2.2 viruses. Influenza and Other Respiratory Viruses, $3,59-62$. 